\title{
DISCUSSION
}

\section{Shear strength parameters from back-analysis of single slips}

\author{
L. D. WESLEY and V. LEELARATNAM, (2001) Géotechnique 51, No. 4, 373-374
}

\section{R. Greco, University of Calabria}

The approach proposed by the authors for deducing shear strength parameters from the analysis of a slide is very interesting, as it is both simple and efficient.

The condition that the safety factor, $F$, is equal to 1 along the actual slip surface gives a relation $c^{\prime}\left(\tan \phi^{\prime}\right)$ represented by curve (a) in Fig. 2, where $c^{\prime}$ decreases with increasing $\tan \phi^{\prime}$. To determine unequivocally the pair $\left(c^{\prime}, \tan \phi^{\prime}\right)$ of the operative values of the shear strength parameters, the authors introduce a further condition by assuming that the actual slip surface is also a critical slip surface. A similar approach was also used by the writer (Greco, 1986), based on the observation that the critical slip surface becomes deeper with increasing cohesion or decreasing friction angle, and becomes shallower with increasing friction angle or decreasing cohesion. This consideration means that an identical critical slip surface can be obtained with different pairs of $\left(c^{\prime}, \tan \phi^{\prime}\right)$. The set of these pairs (connected by the condition $F=\min )$ is represented by a curve of $c^{\prime}\left(\tan \phi^{\prime}\right)$, for which $c^{\prime}$ increases with $\tan \phi^{\prime}$. The intersection of this curve and that relating to the condition $F=1$ gives clearly and unequivocally the operative values of the shear strength parameters acting on the actual slip surface (Fig. 6).

Therefore the method proposed by the authors in the three presented procedures is certainly efficient for identifying operative values of $c^{\prime}$ and $\phi^{\prime}$. Because of the hypothesis concerning the circularity of the slip surfaces, critical slip surfaces can be obtained using the grid method, which makes this approach easier than that of the writer, which, formulated for general slip surfaces, requires a more sophisticated minimisation technique. The basic problem of these approaches lies in the assumption that the actual slip surface is also a theoretical slip surface. In fact, owing to simplifications of analysis, idealisations of soil properties and lack of information, actual slip surfaces can differ appreciably from theoretical critical slip surfaces.

For these cases another method proposed by the writer (Greco, 1996) can be used, in which the operative values of $c^{\prime}$ and $\phi^{\prime}$ are obtained by minimising the area between the

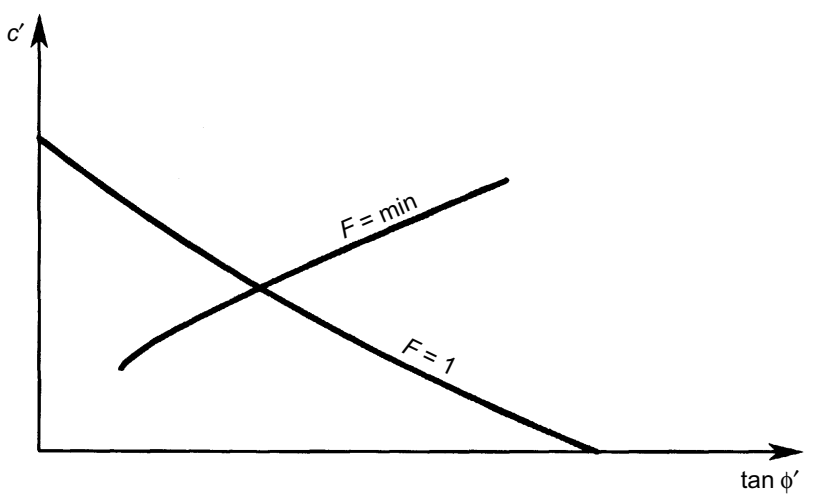

Fig. 6. Determination of the values of $c^{\prime}$ and $\phi^{\prime}$ mobilised at failure using the pair of conditions $F=1$ and $F=\min$. actual slip surface and the critical one. However, each step of this minimisation consists in a further minimisation to search for a critical slip surface. Consequently, the backanalysis is tackled by means of a sequence of minimisation procedures, and the method is not easy to use, in contrast to the authors' proposed method.

The method proposed by Saito (1980) is certainly easy, and no less efficient. Saito proposed back-analysing two further potential slip surfaces, the first external and the second internal with respect to the actual one. The curves of $c^{\prime}\left(\tan \phi^{\prime}\right)$ relating to these two slip surfaces intersect the curve relating to the actual slip surface at two points, between which the operative values of $c^{\prime}$ and $\phi^{\prime}$ fail.

An alternative procedure for Saito's method consists in analysing the two potential slip surfaces (the internal surface and the external one) with the pairs of values $\left(\tan \phi^{\prime}, c^{\prime}\right)$ obtained in the back-analysis of the actual slip surface. Two curves are thus obtained, in which $F$ is expressed in terms of $c^{\prime}$ or $\tan \phi^{\prime}$. The intersection of these curves, if it occurs at $F=1$, gives unequivocally the operative values of $c^{\prime}$ and $\tan \phi^{\prime}$ along the actual slip surface (as in Fig. 7(b), which refers to the problem in Fig. 7(a)).

Saito's method has some important advantages: first, it does not require the actual slip surface to be also a theoretical slip surface, and, second, only two potential slip surfaces are analysed, in addition to the actual one.

However, when using this method the two potential slip surfaces (internal and external) have to be carefully selected. If they are too far from the actual surface the solution can be false (this occurs at a point where $F$ is not equal to 1). By contrast, if they are too close to the actual surface, the differences in $F$ are insignificant. For example, in the case shown in Fig. 7(a) the two potential slip surfaces, related to the curves in Fig. 7(b), differ from the actual surface by $0.1 \mathrm{~m}$ in the radius length.

In the light of the discussion above, the writer believes that, although the method proposed by the authors is an important contribution to the back-analysis of failed slopes, in general Saito's method, which does not require criticality of the actual slip surface, is still a better practical procedure for estimating the shear strength parameter values.

\section{Author's reply}

I am replying to this discussion on my own behalf (as the lead author of the note). I am sorry to say I have lost contact with my fellow author since he left New Zealand to work in south-east Asia.

I am very grateful to Dr Greco for his comments, and for drawing my attention both to his own work and to that of Saito (1980), which I had not previously been aware of. I am sorry that I did not acknowledge the work of both authors in the original note.

I am in general agreement with the points made by $\mathrm{Dr}$ Greco, and in particular that the method of Saito is very attractive as it is simple and easy to use. However, I have reservations on two points.

The first point relates to the statement that 'it [Saito's method] does not require the actual slip surface to be also a 


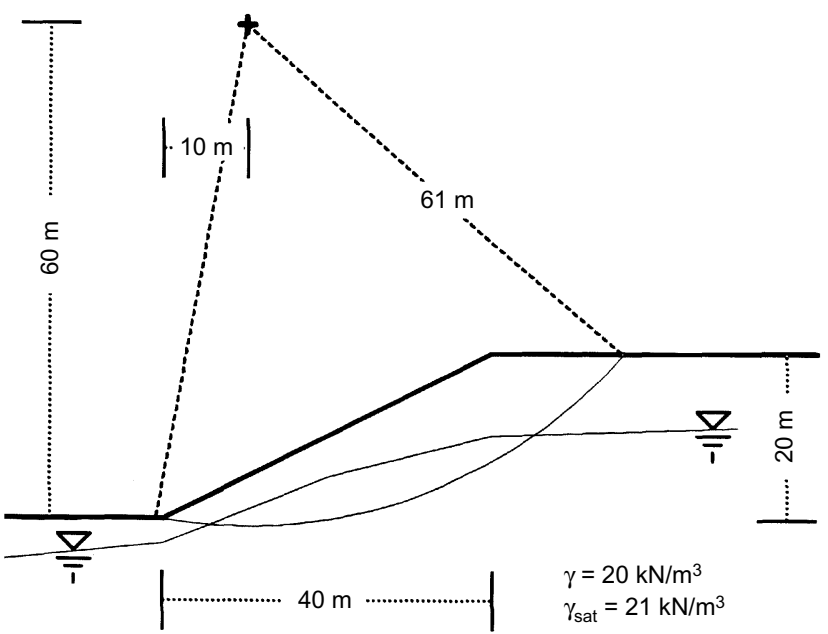

(a)

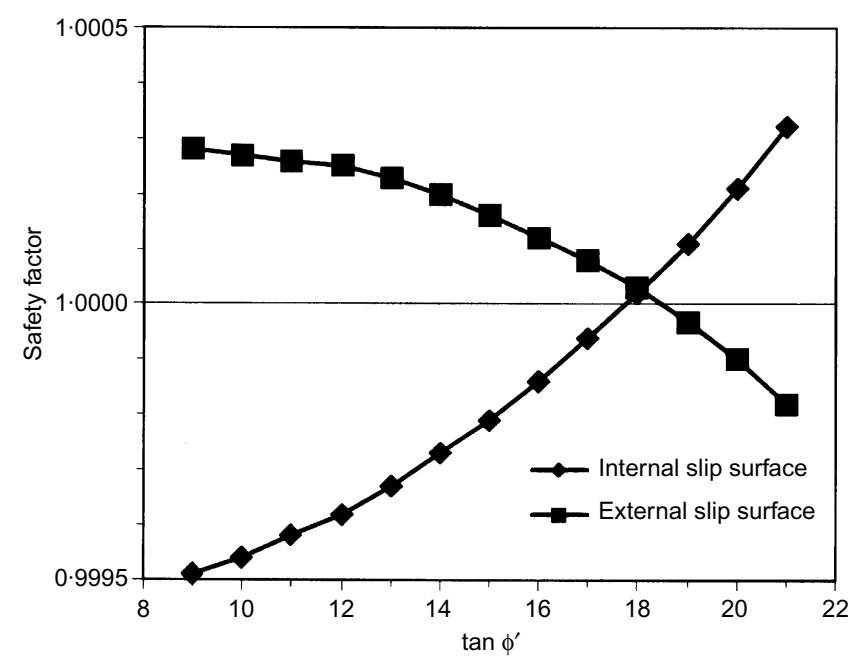

(b)

Fig. 7. (a) Cross-section of a failed slope; (b) graph for determining operative values of $c^{\prime}$ and $\phi^{\prime}$ mobilised at failure

\section{DISCUSSION}

theoretical slip surface'. I presume that here 'theoretical slip surface' means the theoretical critical slip surface in the slope, and if this is the case I am uncertain whether the statement is true. The graphs in Fig. 7(b) show values of safety factor (SF) above and below unity for the two additional trial circles analysed (the internal and external circles). The safety factors above or below unity occur because a range of possible combinations of $c^{\prime}$ and $\tan \phi^{\prime}$ are being tested, only one of which is correct. This procedure thus involves an inherent assumption that the true values of $c^{\prime}$ and $\tan \phi^{\prime}$ on the actual slip plane also apply throughout the rest of the slope, and will give safety factor values greater than unity on any failure surface other than the critical one. It seems to me therefore that this method (along with the authors' methods) is valid only if the slope is homogeneous, in which case the actual slip surface is also the theoretical critical one.

The second point relates to the interpretation put on the two graphs in Fig. 7(b), that the point at which the graphs cross the $\mathrm{SF}=1$ line corresponds to the correct values of $c^{\prime}$ and $\tan \phi^{\prime}$. There can be only one circle having a safety factor of unity with the true values of $c^{\prime}$ and $\tan \phi^{\prime}$ - the critical circle. On any other circle the safety factor will be greater than unity, so that the point at which the graphs in Fig. 7(b) cross the SF $=1$ line does not correspond exactly to the true values of $c^{\prime}$ and $\tan \phi^{\prime}$. However, if circles are taken very close to the failure circle, as advocated by $\mathrm{Dr}$ Greco, this may not be a significant issue.

I should not like to be too categorical about these points, and will be very happy if Dr Greco is given the opportunity to respond to my comments.

\section{REFERENCES}

Greco, V. R. (1986). Valutazione dei parametri di resistenza a taglio dall'analisi di frane. XVI Convegno Italiano di Geotecnica, Bologna 3, 225-230.

Greco, V. R. (1996). Back-analysis of failed slopes. Proceedings of the international conference on landslides (ed. D. H. Bell), Vol. 1, pp. 435-440. Rotterdam: Balkema.

Saito, M. (1980). Reverse calculation method to obtain $c$ and $\phi$ on a slip surface. Proc. 3rd Int. Symp. on Landslides, New Delhi $\mathbf{1}$, 281-284. 